\title{
Diagnostic Efficacy of CCTA And CT-FFR Based On Risk Factors For Myocardial Ischemia
}

\author{
Yongguang Gao \\ Xuzhou Central Hospital \\ Yibing Shi ( 1297230727@qq.com ) \\ Xuzhou Central Hospital \\ Ping Xia \\ Xuzhou Central Hospital \\ Jinyao Zhang \\ Beijing Keyaark Medical Technology Co., Ltd \\ Yufei Fu \\ Xuzhou Central Hospital \\ Yayong Huang \\ Xuzhou Central Hospital \\ Yuanshun Xu \\ Xuzhou Central Hospital \\ Gutao Li \\ Bengbu Medical College
}

\section{Research article}

Keywords: CCTA, CT-FFR, Myocardial ischemia, Fractional flow reserve.

Posted Date: October 11th, 2021

DOI: https://doi.org/10.21203/rs.3.rs-956339/v1

License: (c) (i) This work is licensed under a Creative Commons Attribution 4.0 International License. Read Full License 


\section{Abstract}

Background: Coronary artery CCTA can observe the degree of coronary artery stenosis and FFR can evaluate the physiological function of coronary artery. However, noninvasive imaging examination that can both observe the above two methods at the same time has not yet been elucidated.

Objective: To investigate the diagnostic efficacy of coronary computed tomography angiography (CCTA) and computed tomography-derived fractional flow reserve (CT-FFR) based on different risk factors for myocardial ischemia.

Methods: Patients undergoing CCTA in our hospital from August 18, 2020 to April 28, 2021 were randomly selected, and the data were subjected to CT-FFR analysis. Vascular characteristics were measured, including total plaque volume, calcified plaque volume, non-calcified plaque volume, plaque length, and lumen stenosis, and the patients were categorized into a non-ischemia group (FFR $>0.8)$ and an ischemia group (FFR $\leq$ 0.8). Plaque characteristics were compared between the two groups, and logistic regression analysis was employed to explore the correlations between plaque characteristics and ischemic lesions.

Results: From a total of 122 patients enrolled in the study, there were 218 vascular branches with FFR>0.8 and 174 vascular branches with $\mathrm{FFR} \leq 0.8$. There were significant group differences in total plaque volume, calcified plaque volume, plaque length, and lumen stenosis $>50 \%$ (n). The obtained data were as follows: non-ischemic group 10.57 (4.80,259.65), ischemic group 14.87 (3.39, 424.45), $Z=9.772, p=0.002$, non-ischemic group $10.57(0,168.77)$, ischemic group $14.87(0,191.00), Z=2.503 \rrbracket p \leq 0.001)$, non-ischemic group 8.17 $(37.05,40.53)$, ischemic group $8.38(56.66,86.47), Z=5.923, p=0.016$, and lumen stenosis $>50 \%$, non-ischemic group 46 , ischemic group $90, x^{2}=14.77 \rrbracket$ $p \leq 0.001$. The regression analysis results indicated that total plaque volume, calcified plaque volume, plaque length and lumen stenosis $>50 \%$ were risk factors for myocardial ischemia, with ORs and $p$ values of $(2.311, p=0.002),(1.021, p=0.004),(2.159, p<0.001)$, and $(0.181$, $p<0.001)$, respectively.

Conclusion: Total plaque volume, calcified plaque volume, plaque length and lumen stenosis $>50 \%$ are predictors for myocardial ischemia. Coronary artery CCTA combined with CT-FFR could simultaneously observe the anatomical stenosis and evaluate myocardial blood supply at the functional level. Thus, myocardial ischemia could be better diagnosed.

\section{Background}

China has an immense population and a substantial number of people with coronary atherosclerosis. Coronary computed tomography angiography (CCTA) is a first-line imaging tool to exclude and diagnose coronary heart disease, and is characterized by low cost, simplicity of operation, and high negative predictive value. CCTA has been used extensively as a non-invasive approach to determine coronary heart disease in China.

Invasive coronary angiography (ICA) and CCTA can identify anatomical stenosis, but cannot functionally evaluate myocardial blood supply. Coronary artery-based fractional flow reserve (FFR) is able to evaluate the physiological function of coronary arteries. However, it is difficult to promote widely due to its invasive nature (e.g., possible destruction of plaque stability during surgery, or vascular wall damage), high cost, increased surgical risk, and adenosine side effects. Furthermore, CT-derived FFR (CT-FFR) is emerging as a non-invasive method to solve these problems, which can both identify vascular anatomical stenosis and evaluate myocardial blood supply from a functional perspective.

With the rapid progression of $\mathrm{Al}$ and electronic computers, non-invasive CT-FFR has developed rapidly in recent years. Previous studies have confirmed that CT-FFR is highly accurate in diagnosing coronary artery stenosis [1, 2]. Moreover, CT-FFR was highly correlated with traditional ICA-FFR, since the relevant outcomes can be calculated using the basic governing equation of fluid mechanics, namely the Navier-Stokes equation which assumes that blood is an incompressible Newtonian fluid with constant density and viscosity [3]. In 2019, according to Guidelines for the Diagnosis and Management of Chronic Coronary Syndromes released by the European Society of Cardiology, non-invasive functional imaging techniques have been recommended to be first-line examination approaches (Classification I recommendation) for clinical diagnosis and treatment of patients with suspected or known coronary heart disease [4].

An ICA-based diagnosis alone is not sufficient in the case of partial diseased vascular stenosis $>50 \%$. The reason for this is that some diseased blood vessels may form collaterals, resulting in a certain compensatory effect, and ICA analysis cannot truly detect the myocardial ischemia dominated by the diseased blood vessels, leading to a mismatch between stenosis severity and myocardial ischemia [5]. Studies have demonstrated that for patients who are considered for stent implantation after ICA examination, approximately $32 \%$ do not require stent intervention [6]. Therefore, the search for a better tool to judge functional coronary artery stenosis has become a current research hotspot. 


\section{Objectives}

In view of a substantial number of people with coronary atherosclerosis in China, the present study aims to investigate the influences of different risk factors for myocardial ischemia on CT-FFR using CCTA, so as to allow more atherosclerosis patients to receive the benefits of imaging examination, such as low cost and non-invasiveness.

\section{Methods}

\section{General data}

The clinical data of 132 patients diagnosed with known or suspected coronary heart disease after undergoing CCTA were retrospectively analyzed by CT-FFR. The inclusion criteria were as follows: a) patients with qualified CCTA image quality, and b) those with valid data following CT-FFR software calculation and feedback. Patients were excluded for the following: $a$ ) having received a stent implantation ( $\mathrm{n}=4$ ), b) having undergone coronary artery bypass grafting surgery $(n=4)$, and $c$ ) having data that could not be identified by DICOM software ( $n=2)$. A total of 122 patients were enrolled, with 396 diseased blood vessels, including 218 vascular branches with FFR $>0.8$ and 174 vascular branches with FFR $\leq 0.8,168$ anterior descending branches, 56 circumflex branches, 120 right coronary arteries, 16 first diagonal branches, 10 second diagonal branches, 10 blunt marginal branches, and 12 intermediate branches, which were subjected to CT-FFR plaque measurement and evaluation. Among the 122 patients, there were 72 males and 50 females with ages ranging from 32 to 82 (59.9 \pm 8.6 ) years old.

Research methods

All patients received conventional CCTA according to the Society of Cardiovascular Computed Tomography Guidelines. In this study, two CT scanners were used, a 320-row CT (Aquilion One, Toshiba, Japan) and a 640-row CT (Aquilion ViSION, Toshiba, Japan). A double-headed high-pressure syringe was utilized to inject $40-60 \mathrm{~mL}$ of the non-ionic contrast agent iopromide (containing $370 \mathrm{mg} / \mathrm{mL}$ iodine, Bayer Schering Pharma) at a flow rate of $4.0-5.0 \mathrm{~mL} / \mathrm{s}$, and then $30 \mathrm{~mL}$ of normal saline at the same flow rate. Patients were in the supine position during the scan and were routinely monitored by electrocardiograph (ECG). The scan range was $1.0 \mathrm{~cm}$ below the carina to $1.5 \mathrm{~cm}$ from the inferior margin of the heart. No patients received nitroglycerin or $\beta$-blockers. CT scan parameters included: a) collimation: $320 \times 0.500$, reconstruction thickness: $0.500 \mathrm{~mm}$, tube rotation speed: $0.35 \mathrm{~s}$, tube voltage: $100 \mathrm{kV}$ or $120 \mathrm{kV}$, tube current: $200-400 \mathrm{~mA}$, and detector collimation: $160-320 \times 0.5 \mathrm{~mm}$, and b) Aquilion ViSION, scan parameters: collimation: $160-320 \times 0.5 \mathrm{~mm}$, reconstruction thickness: $0.500 \mathrm{~mm}$, tube rotation speed: $0.275 \mathrm{~s} / \mathrm{r}$, tube voltage: $100 \mathrm{kV}$ (smart), and tube current: $200-400 \mathrm{~mA}$. All scanning devices adopt prospective ECG gating for image acquisition, followed by optimal image quality reconstruction. The images meeting the diagnostic requirement were transferred to the post-processing workstation for subsequent analysis.

CCTA images of all enrolled patients were uploaded to the core laboratory for blind reading. Lumen stenosis on CCTA images was diagnosed by two readers with more than 5 years of working experience in coronary artery CT using the visual diameter method.

Non-invasive CT-FFR measurement was carried out as follows: the standard DICOM format CT data of all patients were transferred to the professional CT-FFR measurement software (China Keya Medical Corporation-Deep Pulse Score DEEPVESSEL FFR, China), and CTFFR measurement was performed by a dedicated operator blinded to the results of other examinations. Using plague segmentation and a quantitative analysis model based on deep learning algorithms, artificially labeled plaque data were processed by automatic detection and segmentation model training, and plaque volume, plaque composition, plaque length, and remodeling index of the segmentation results were calculated.

\section{Statistical analysis}

SPSS 17.0 software was used for statistical analysis. Continuous variables were tested to ensure normal distribution. Normally distributed data were expressed by mean \pm standard deviation $(\chi \pm s)$, and those not conforming to a normal distribution were represented by the median (upper and lower quartiles) [M (Q1, Q2)]. Categorical data were expressed by frequency (positive remodeling, stenosis $>50 \%)$, and measurement data (total plaque volume, calcified plaque volume, non-calcified plaque volume, and plaque length) were non-normally distributed. According to the FFR value, the patients were grouped into a non-ischemia group (FFR $>0.8)$ and an ischemia group (FFR $\leq 0.8$ ), and plaque characteristics were compared between the two groups. Logistic regression analysis was used to confirm the correlations between plaque characteristics and ischemic lesions. Group differences in normally distributed variables were analyzed using independentsamples $t$-tests, and Rank sum tests were utilized for non-normal variable. Data analysis was based on the number of blood vessels. $p<0.05$ was used to indicate statistical significance. 


\section{Results}

Comparison of clinical data

A total of 122 patients were enrolled in this study, including 66 cases with FFR $>0.8$ and 56 cases with FFR $\leq 0.8$. Regarding comorbidities, 60 patients had hypertension, 38 had diabetes, 34 had hyperlipidemia, 42 were smokers, and 48 were excessive drinkers. Group differences in the general data were not statistically significant (Table 1).

Table 1

\begin{tabular}{|c|c|c|c|c|c|c|c|c|c|c|c|c|c|c|c|c|}
\hline \multirow[b]{2}{*}{ Group } & \multirow[b]{2}{*}{$\mathrm{n}$} & \multirow[b]{2}{*}{$\begin{array}{l}\text { Age }(Y) \otimes \\
X \pm S\end{array}$} & \multicolumn{3}{|c|}{ Gender } & \multirow[b]{2}{*}{$\begin{array}{l}\text { BMI } \\
\mathrm{kg} / \mathrm{m}^{2 \rrbracket} x \pm s\end{array}$} & \multicolumn{2}{|c|}{ Hypertension } & \multicolumn{2}{|c|}{ Diabetes } & \multicolumn{2}{|c|}{ Hyperlipidemia } & \multicolumn{2}{|c|}{ Smoking } & \multicolumn{2}{|c|}{ Drinking } \\
\hline & & & & Male & Female & & Yes & No & Yes & No & Yes & No & Yes & No & Yes & No \\
\hline $\mathrm{FFR}>0.8$ & 66 & $59.7 \pm 9.1$ & & 40 & 26 & $26.3 \pm 3.9$ & 32 & 34 & 16 & 50 & 10 & 56 & 18 & 48 & 26 & 40 \\
\hline$F F R \leq 0.8$ & 56 & $61.3 \pm 7.7$ & & 32 & 24 & $24.9 \pm 3.9$ & 28 & 28 & 22 & 34 & 24 & 32 & 26 & 30 & 22 & 34 \\
\hline$x^{2}(t)$ & & $-0.437^{a}$ & 0 & & & $0.925^{a}$ & 0 & & 0 & & 0.872 & & 0.019 & & 0 & \\
\hline$p$ & & 0.665 & 1 & & & 0.391 & 1 & & 1 & & 0.35 & & 0.891 & & 1 & \\
\hline
\end{tabular}

Plaque characteristics were compared between the ischemia and non-ischemia groups, and statistically significant differences were observed for total plaque volume, calcified plaque volume, plaque length, and lumen stenosis $>50 \%(p<0.05)$. Patients with stenosis $>50 \%$ were common, showing a statistically significant difference. However, non-calcified plaque volume and positive remodeling were not statistically significant between the two groups (Table 2).

Table 2

\begin{tabular}{|c|c|c|c|c|c|c|c|}
\hline Group & $\begin{array}{l}\text { Diseased vessels } \\
\text { (branches) }\end{array}$ & $\begin{array}{l}\text { Total volume } \\
\text { of plaques }\end{array}$ & $\begin{array}{l}\text { Volume of } \\
\text { calcified plaques }\end{array}$ & $\begin{array}{l}\text { Volume of non- } \\
\text { calcified plaques }\end{array}$ & $\begin{array}{l}\text { Lesion } \\
\text { length }\end{array}$ & $\begin{array}{l}\text { Positive } \\
\text { remodeling } \\
\text { (n) }\end{array}$ & $\begin{array}{l}\text { Stenosis } \\
>50 \%(n)\end{array}$ \\
\hline \multirow[t]{2}{*}{$F F R>0.8$} & 218 & 10.57 & 10.57 & 32.89 & 8.17 & 202 & 46 \\
\hline & & $(4.80,259.65)$ & $(0,168.77)$ & $(4.35,192.41)$ & $\begin{array}{l}\text { (37.05, } \\
40.53)\end{array}$ & & \\
\hline \multirow[t]{2}{*}{$F F R \leq 0.8$} & 174 & 14.87 & 14.87 & 43.53 & 8.38 & 164 & 90 \\
\hline & & $(3.39,424.45)$ & $(0,191.00)$ & $(3.25,374.35)$ & $\begin{array}{l}(56.66 \\
86.47)\end{array}$ & & \\
\hline Statistics & & $9.772^{a}$ & $2.503^{a}$ & $11.204^{a}$ & $5.923^{b}$ & $0^{b}$ & $14.77^{b}$ \\
\hline$p$ & & 0.002 & $<0.001$ & 0.115 & 0.016 & 1 & $<0.001$ \\
\hline
\end{tabular}

The results of logistic univariate regression analysis indicated that total plaque volume, calcified plaque volume, plaque length, and lumen stenosis $>50 \%$ could induce myocardial ischemia, with odds ratios (OR) of $2.161,1.041,1.322,0.233$, and $p$ values of $0.002,0.001,<0.001$, $<0.001$, respectively. Risk factors showing significant differences $(p<0.05)$ were incorporated into a multivariate regression analysis, which yielded ORs of $2.311,1.021,2.159,0.181$, and $p$ values of $0.002,0.004,0.002,<0.001$, respectively. Among these, lumen stenosis $>50 \%$ was the first major factor, and the OR was 0.181, $p<0.001$ (Table 3). 
Table 3

\begin{tabular}{|lrlllll|}
\hline & \multicolumn{3}{c}{ Univariate analysis } & \multicolumn{3}{c|}{ Multivariate analysis } \\
\hline Index & $\mathrm{OR}(95 \% \mathrm{Cl})$ & & $p$ & $\mathrm{OR}(95 \% \mathrm{Cl})$ & $p$ \\
\hline Total volume of plaques & 2.161 & $1.496-4.689$ & 0.002 & 2.311 & $2.117-5.854$ & 0.002 \\
\hline Volume of calcified plaques & 1.041 & $1.010-1.073$ & 0.001 & 1.021 & $0.989-1.055$ & 0.004 \\
\hline Volume of non-calcified plaques & 0.996 & $0.990-1.002$ & 0.812 & - & - & - \\
\hline Lesion length & 1.322 & $1.116-1.121$ & $<0.001$ & 2.159 & $1.482-3.385$ & 0.002 \\
\hline Positive remodeling & 1.175 & $0.357-3.864$ & 0.79 & - & - & - \\
\hline Medium-severe stenosis & 0.233 & $0.094-0.577$ & $<0.001$ & 0.181 & $0.057-0.578$ & $<0.001$ \\
\hline Note: "-" represents no data. & & & & & & \\
\hline
\end{tabular}

\section{Discussion}

According to the results of CCTA and CT-FFR, the patients were categorized into non-ischemia group (FFR >0.8) and ischemia group (FFR $\leq 0.8$ ). Plaque characteristics were compared between the two groups, and the results showed that the differences in total plaque volume, calcified plaque volume, plaque length, and lumen stenosis $>50 \%$ were significantly different. Moreover, different risk factors were investigated by logistic regression analysis, and the results indicated that total plaque volume, calcified plaque volume, plaque length, and lumen stenosis $>50 \%$ were influencing factors for myocardial ischemia, especially lumen stenosis $>50 \%$, with an $0 \mathrm{R}$ of 0.181 ( $p<0.001$ ). Therefore, CCTA combined with CT-FFR has high diagnostic efficacy for coronary heart disease. A routine test can be used to observe anatomical stenosis and functional test. It is noninvasive and low-cost, which reduces the burden for patients.

Multiple studies demonstrated that CT-FFR has a higher diagnostic accuracy for coronary heart disease than traditional CCTA [7-9]. Studies have confirmed that about half of the patients with significant coronary stenosis on CCTA have lumen stenosis $>50 \%$, but without myocardial ischemia or only small patchy perfusion ischemia as detected by radionuclide imaging [10], and some patients receiving stent implantation have no significant symptom improvement after surgery [11]. In this study, the results also showed that the patients with lumen stenosis $>50 \%$ had CT-FFR $>0.8$, without obvious clinical symptoms. The reason for this may be that the CT-FFR value was not decreased due to collateral formation, while patients with mild stenosis ( $<50 \%)$ may suffer from myocardial ischemia, indicating that the anatomical stenosis does not match the actual functional ischemia. In most studies, FFR $\leq 0.8$ is selected as the threshold, and FFR is considered as a guide [12]. When FFR $>0.8$, there is no myocardial ischemia, so the therapeutic effect of medication is better. However, when FFR $<0.8$, suggesting the presence of myocardial ischemia, the probability of the end-point event of patients with PCI treatment is significantly lower than that of patients with drug treatment [13]. Moreover, a portion of patients with CT-FFR slightly greater than 0.8 can also have more obvious clinical symptoms. This may be due to the fact that CT-FFR is a computer-derived data product, which has a higher correlation compared with the real-time data of invasive FFR but has differences, especially for patients with cut-off values.

The Chinese Guidelines for Percutaneous Coronary Intervention (2016) recommends therapeutic intervention when coronary artery stenosis is less than $90 \%$ and there is evidence of myocardial ischemia, or when FFR is less than 0.8 , thereby greatly reducing the stent implantation rate and avoiding excessive medical treatment [14]. For patients with myocardial ischemia, surgical revascularization is the first choice by clinicians $[15,16]$. By observing patient's clinical symptoms and evaluating stenosis severity, FFR-guided CABG has a significantly lower mortality rate or probability of myocardial infarction after 6 years over ICA-guided coronary artery bypass grafting surgery [17].

Different types of atherosclerotic plaques have different stability. The pathogenic process is the vascular intima with reactive hyperplasia evolving into fibrous and calcified plaques [18]. It is generally believed acute cardiovascular events mainly occur due to the rupture or erosion of high-risk plaques, and lumen stenosis caused by fibrous or calcified plaques can induce chronic myocardial ischemia [19]. Consistent with previous studies, risk factors such as positive remodeling, napkin-ring sign, punctate calcification, and low-density plaque are characteristic of high-risk plaques, which can increase the occurrence of acute coronary syndromes. In contrast, simple calcified and fibrous plaques can only induce myocardial ischemia due to their stable nature (i.e., not easy to rupture).

To date, CT-FFR research has made considerable progress. However, CCTA image-based CT-FFR still has many uncertain factors for blood flow and arterial blood analysis, and the reasons are as follows: A) Different companies have different algorithms and varying calculation times. Although the processing time has shortened in recent years, it still takes a few hours at the fastest speed. B) The research data are relatively homogeneous. The main purpose of CCTA is to screen a large range of people, but there is no relevant evidence for patients with 
acute coronary syndrome. C) The CT-FFR algorithm simulates the filling state of blood vessels, which have individual differences, such as elasticity. The same model and formula will reduce accuracy, but for patients with microcirculation, the obtained CT-FFR value is too large [20]. D) For evaluating patients with diffuse calcification, severe calcification will inevitably cause artifacts due to the limitation of CCTA images, and the data obtained will be lower.

In recent years, with the rapid development of CT-FFR, numerous experiments and evidence-based medicine verified that the technique has become the "gatekeeper" of ICA examination. ICA can characterize lumen stenosis, but cannot detect physiological ischemia. As a result, clinically significant lesions cannot be accurately treated and medical resources are wasted, while real lesions leading to myocardial ischemia go untreated [21].

There were many shortcomings in this study. Firstly, FFR was not used as the gold standard, but the accuracy of CT-FFR in diagnosing coronary artery stenosis has been confirmed in many previous studies. Moreover, CT-FFR had high correlation with traditional FFR [1-3], and the recognition was gradually improved. Secondly, this article was a retrospective study with a small sample size, and branch vessels were included in the study, which may lead to statistical biases against previous studies. Thirdly, patients who underwent stent implantation and coronary artery bypass grafting surgery were excluded from this study, so the research population needs to be comprehensively improved in future studies.

\section{Conclusion}

In conclusion, total plaque volume, calcified plaque volume, plaque length, and stenosis lumen $>50 \%$ are risk factors for myocardial ischemia, and CCTA combined with CT-FFR can effectively diagnose myocardial ischemia.

\section{Declarations}

\section{Acknowledgement}

None.

\section{Funding}

The Science and Technology Project of Xuzhou City (KC19176).

\section{Availability of data and materials}

All data generated or analyzed during this study are included in this article.

\section{Authors' contributions}

Gao Yongguang designed this study; Xia Ping, Fu Yufei, and Li Gutao performed the coil localization; Huang Yayong, Xu Yuanshun, and Gao Yongguang collected the patients' data; Zhang Jinyao and Gao Yongguang analyzed these data; Shi Yibing wrote and revised this paper; Final manuscript was approved by all authors.

\section{Ethics approval and consent to participate}

This retrospective study was approved by institutional review board of Xuzhou Central Hospital. The written informed consent was waived due to the retrospective nature. Only the relevant patient provided informed consent for publication of the images in Figure 1-3.

\section{Consent for publication}

The relevant patient provided informed consent for publication of the images in Figure 1-3.

\section{Competing interests}

The authors declare that they have no competing interests.

\section{Author details}

${ }^{1}$ Department of Radiology, Xuzhou Central Hospital, 199 Jiefang Road, Xuzhou, Jiangsu, China.

2 Beijing Keyaark Medical Technology Co., Ltd. 
${ }^{3}$ Graduate School, Bengbu Medical College, 2600 Donghai Avenue, Bengbu, Anhui, China.

\section{References}

1. Tesche C, Vliegenthart R, Duguay TM, et al. Coronary computed tomographic angiography-derived fractional flow reserve for therapeutic decision making. Am J Cardiol. 2017;120:2121-7.

2. Tesche C, De Cecco CN, Baumann S, et al. Coronary CT angiography-derived fractional flow reserve: machine learning algorithm versus computational fluid dynamics modeling. Radiology. 2018;288:64-72.

3. Lee JM, Choi G, Koo BK, et al. Identification of high-risk plaques destined to cause acute coronary syndrome using coronary computed tomographic angiography and computational fluid dynamics. JACC-Cardiovasc Imag. 2019;12:1032-43.

4. Knuuti J, Wijns W, Saraste A, et al. 2019 ESC Guidelines for the diagnosis and management of chronic coronary syndromes. Eur Heart J. 2020;41:407-77.

5. Foy AJ, Dhruva SS, Peterson B. et a1. Coronary computed tomography angiography vs functional stress testing for patients with suspected coronary artery disease a systematic review and meta-analysis. Jama Intern Med. 2017;177:1623-31.

6. Sant'Anna FM, Silva EE, Batista LA, et a1. Influence of routine assessment of fractional flow reserve on decision making during coronary interventions. Am J Cardiol. 2007; 99: 504-508.

7. Koo BK, Erglis A, Doh JH, et al. Diagnosis of ischemia-causing coronary stenoses by noninvasive fractional flow reserve computed from coronary computed tomographic angiograms results from the prospective multicenter DISCOVER-FLOW (diagnosis of ischemia-causing stenoses obtained via noninvasive fractional flow reserve) study. J Am Coll Cardiol. 2011;58:1989-97.

8. Min JK, Leipsic J, Pencina MJ, et al. Diagnostic accuracy of fractional flow reserve from anatomic CT angiography. JAMA-J Am Med Assoc. 2012;308:1237-45.

9. Douglas PS, Pontone G, Hlatky MA, et al. Clinical outcomes of fractional flow reserve by computed tomographic angiography-guided diagnostic strategies vs. usual care in patients with suspected coronary artery disease: the prospective longitudinal trial of FFRCT: outcome and resource impacts study. Eur Heart J. 2015;36:3359-67.

10. Boden WE, O'Rourke RA, Teo KK, et al. Optimal medical therapy with or without PCI for stable coronary disease. N Engl J Med. 2007;356:1503-16.

11. Zimmermann FM, Ferrara A, Johnson NP. Deferral vs. performance of percutaneous coronary intervention of functionally non-significant coronary stenosis: 15-year follow-up of the DEFER trial. Eur Heart J. 2015;36:3182-8.

12. Norgaard BL, Leipsic J, Gaur S, et al. Diagnostic performance of noninvasive fractional flow reserve derived from coronary computed tomography angiography in suspected coronary artery disease: the NXT trial (analysis of coronary blood flow using CT angiography: next steps). J Am Coll Cardiol. 2014;63:1145-55.

13. De Bruyne B, Fearon WF, Pijls NH. et a1. Fractional flow reserve-guided PCl for stable coronary artery disease. N Engl J Med. 2014;371:1208-17.

14. Li L, Hu X, Tao X, et al. Radiomic features of plaques derived from coronary CT angiography to identify hemodynamically significant coronary stenosis, using invasive FFR as the reference standard. Eur J Radiol. 2021;140:109769.

15. Kolh P, Windecker S, Alfonso F, et al. 2014 ESC/EACTS guidelines on myocardial revascularization. Eur J Cardio-thorac. 2014;46:51792.

16. Patel MR, Calhoon JH, Dehmer GJ, et a1. ACC/AATS/AHA/ASE/ASNC/SCAI/SCCT/STS 2017 appropriate use criteria for coronary revascularization in patients with stable ischemic heart disease. J Am Coll Cardiol. 2017; 69: 2212-2241.

17. Fournier S, Toth GG, De Bruyne B. et a1. Six-year follow-up of fiactional flow reserve-guided versus angiography-guided coronary artery bypass graft surgery. Circ-Cardiovasc Inte. 2018;11:1-7.

18. Otsuka F, Yasuda S, Noguchi T, et al. Pathology of coronary atherosclerosis and thrombosis. Cardiovasc Diagn The. 2016;6:396-408.

19. Yahagi K, Davis HR, Arbustini E, et al. Sex differences in coronary artery disease: pathological observations. Atherosclerosis. 2015;239:260-7.

20. Taylor CA, Fonte TA, Min JK. Computational fluid dynamics applied to cardiac computed tomography for noninvasive quantification of fractional flow reserve scientific basis. J Am Coll Cardiol. 2013;61:2233-41.

21. Koo BK. The present and future of fractional flow reserve. Circ J. 2014;78:1048-54.

\section{Figures}



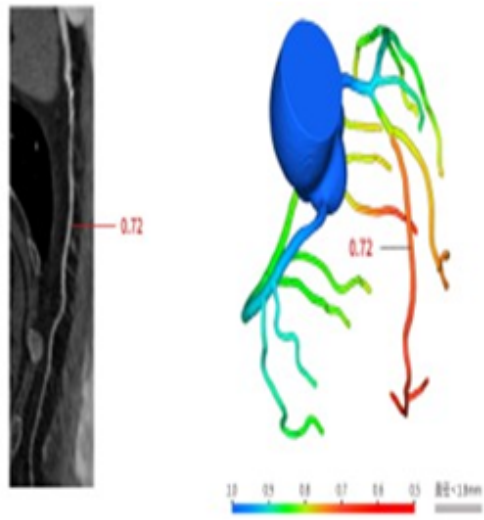

\section{Figure 1}

CT blood flow reserve fraction CT-FFR measurement by automatic analysis software. The FFR value was 0.72 .

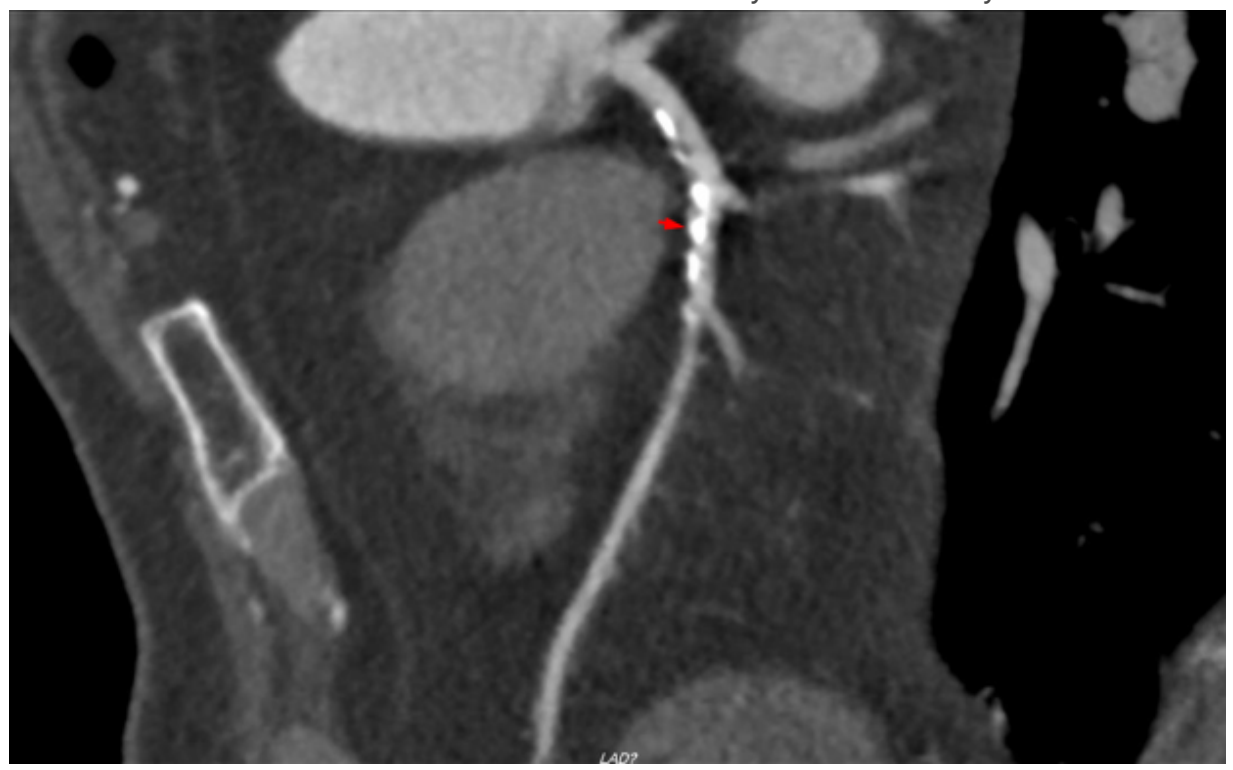

\section{Figure 2}

CCTA and curved planar reconstruction results. An atherosclerotic plaque was indicated by red arrow.

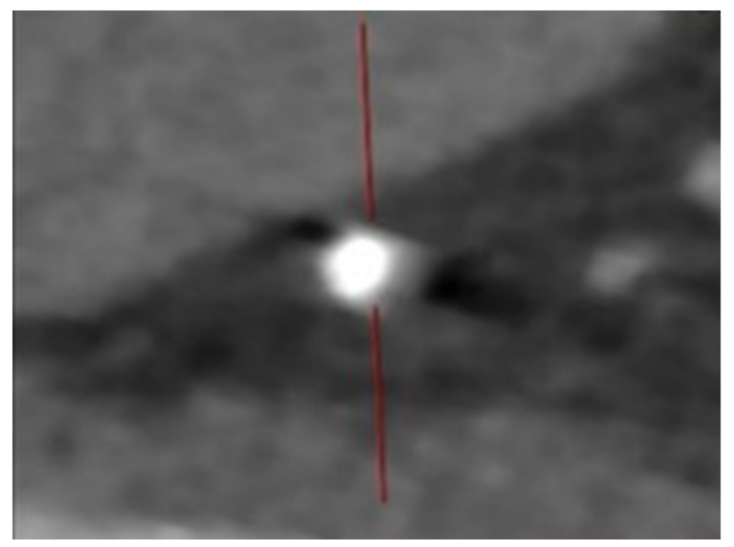

\section{Figure 3}

Cross section of an atherosclerotic plaque with the lumen stenosis $>50 \%$. 